\title{
CT Output Dose Performance-Conventional Approach Verses the Dose Equilibrium Method
}

\author{
Ahmad Albngali ${ }^{*}$, Andy Shearer ${ }^{1}$, Wil van der Putten ${ }^{1,2}$, Brendan Tuohy ${ }^{1,2}$, Niall Colgan ${ }^{1,2}$ \\ ${ }^{1}$ Department of Physics, National University of Ireland, Galway, Ireland \\ ${ }^{2}$ Department of Medical Physics and Clinical Engineering, Galway University Hospitals, Galway, Ireland \\ Email: *A.ALBNGALI1@NUIGALWAY.IE
}

How to cite this paper: Albngali, A., Shearer, A., van der Putten, W., Tuohy, B. and Colgan, N. (2018) CT Output Dose Performance-Conventional Approach Verses the Dose Equilibrium Method. International Journal of Medical Physics, Clinical Engineering and Radiation Oncology, 7, 15-26.

https://doi.org/10.4236/ijmpcero.2018.71002

Received: November 17, 2017

Accepted: January 30, 2018

Published: February 2, 2018

Copyright (c) 2018 by authors and Scientific Research Publishing Inc. This work is licensed under the Creative Commons Attribution International License (CC BY 4.0).

http://creativecommons.org/licenses/by/4.0/

\begin{abstract}
Current output dose measurement in CT is based on (CTDI). The conventional methodology of CT dosimetric performance characterization is not appropriate to modern CT scanners with helical scanning modes, dose modulation, array detectors and multiple slice planes or cone-beam irradiation geometries. AAPM TG 111 report recognizes the shortfall of the CTDI methods and recommends a new technique that more accurately characterizes the dose profile from modern CT scanners, which utilizes a short conventional ion chamber rather than a pencil chamber. We developed and characterize a in-house phantom design using a three separate anatomical regions of clinical scan sequences (Head, chest and abdomen), and determined the equilibrium dose in our dose equilibrium phantom, measured if the attenuation of the beam is the equal to that of CTDI Perspex phantom and compare CTDI dose estimations using a standard pencil chamber to the dose equilibrium phantom measurements. This methodology allows measurements of the accumulated dose for any clinical scan length and allowing measurement of the equilibrium dose. Using the new methodology, we determined that the CTDI approach can underestimate the dose by $25 \%$ to $35 \%$ and all of our dose values from the water phantom and farmer chamber were independently verified with TLD measurements.
\end{abstract}

\section{Keywords}

Computed Tomography, Dixon Method, Farmer Chamber, CT Dosimetry

\section{Introduction}

About 80 million CT exams are performed annually in the United States [1] and $\mathrm{CT}$ is the largest source of medical radiation exposure. It contributes to half of the total exposure from medical applications in the United States [2] [3] and re- 
quires accurate dose quantification for both pediatric and adult patients.

The current method of CT dosimetry is based on the computed tomography dose index concept (CTDI), which represents a single axial rotation of the X-ray tube exposure in a CTDI phantom. It is measured with a $100-\mathrm{mm}$ long pencil ionization chamber (CTDI100) placed in a cylindrical PMMA phantom representing the head and body, respectively. Modern CT scanners with helical scanning modes, dose modulation, array detectors and multiple slice planes or cone-beam irradiation geometries make CTDI unsuitable [4] [5]. The CTDI method is based on two major limitations, firstly the pencil ionization chamber, which is 100 -mm-long and used to collect the dose is not sufficiently long enough to measure the tails of the scattered dose distribution [6], secondly the CTDI phantoms are 14-cm-long, much shorter than the average adult torso, and cannot replicate the scattered radiation that would occur in a typical adult [7].

American association of physics in medicine task group report No. 111 (AAPM TG111) [8] described a new measurement method that would take into account the scatter of modern CT scanners. They recommend using a small volume ion chamber, and a phantom length that allows for dose equilibrium at the location of the chamber [8]. As this type of phantom is not commercially available we aimed to construct and characterize a phantom based on the AAPM TG 111 report and measure the accumulated dose and equilibrium dose using a farmer chamber. Our second objective was to compare the dose estimation of the current CTDI standard and independently verify with TLD's our dose measurements. It's our hypothesis that the $D_{(\mathrm{eq})}$ method correctly estimates the true patient dose

\section{Materials and Methods}

\subsection{Materials}

\subsubsection{Dose Equilibrium Phantom}

An in-house water based phantom was developed based on the work of Dixon et al., [8] [9] referred to from this point as the dose equilibrium $D_{(\mathrm{eq})}$ phantom. $D_{(\mathrm{eq})}$ phantom was designed and constructed in NUI Galway Figure 1. It is $32-\mathrm{cm}$ in diameter and $50 \mathrm{~cm}$ in length. The $D_{(\mathrm{eq})}$ phantom is constructed with a center hole and four peripheral holes. The holes are filled with blanks when not

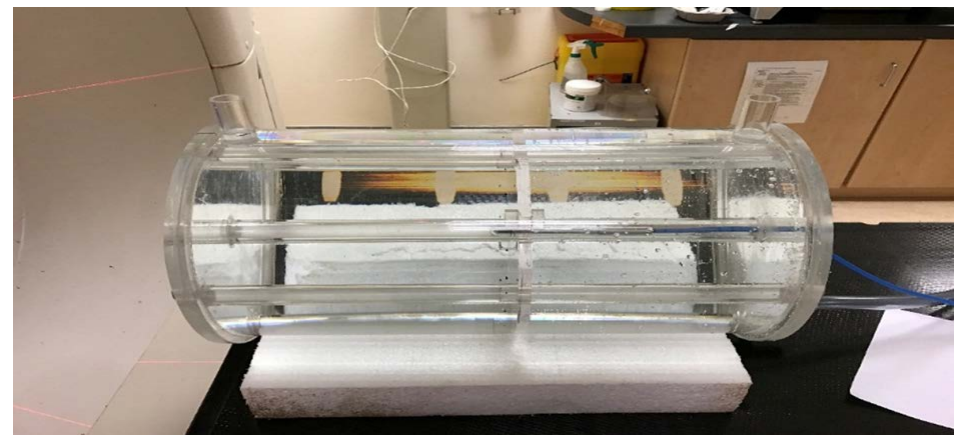

Figure 1. $D_{\text {(eq) }}$ phantom with ion chamber fitted in the center. 
in use. It is designed to be transported empty, and once placed on the table, it can be quickly filled or emptied with a small pump operating from a room sink as a reservoir. This phantom size was chosen in order to represent the attenuation and absorption characteristics of the average size adult body. The material composition of the phantom was based on IAEA TRS 277 [10].

\subsubsection{Perspex Phantom}

The CTDI phantom was a commercial parties from West Physics company. it consisted of two PMMA cylinders: one cylinder represents the head $(160 \mathrm{~mm}$ diameter) and the other represents the body ( $320 \mathrm{~mm}$ diameter). The length of each cylinder is $140 \mathrm{~mm}$. Each cylinder contains holes large enough to accept a pencil chamber and are filled with Perspex blanks when not in use.

\subsubsection{Computed Tomography Scan, Ionization Chambers and TLDs}

A Philips, AcQsim CT was used for these measurements. Dose measurements were taken using Farmer chamber (PTW type 30013) Figure 2 and pencil chamber (Unfors xitm). Both are ionization-based chambers. The Farmer chamber collection volume is $0.6 \mathrm{~cm}^{3}$. It was calibrated by the National Standards of the German National Laboratory. A PC electrometer from Sun Nuclear was used for the Farmer chamber, configured with a cable long enough to allow the electrometer to operate outside the scatter-radiation field, in order to avoid inducing extraneous currents in the electrometer. The PC electrometer provides a bias voltage of \pm 300 Volts, and it has sensitivity and a leakage current consistent with the farmer ionization chamber volume and dose rate.

The pencil chamber is $10 \mathrm{~cm}$ long and was calibrated by Unfors RaySafe. Dose verification was performed independently using thermoluminescent dosimeters (TLDs 100H) Figure 3 which were independently check for accuracy.

\subsection{Methods}

\subsubsection{Beam Attenuation in the $\left(D_{(\mathrm{eq})}\right)$ Phantom and CTDI Phantom}

The dose output from one single slice in the middle of the $D_{(\mathrm{eq})}$ phantom and Perspex phantom by using the pencil chamber was used to validate the dose attenuation from $D_{\text {(eq) }}$ phantom. For this work, the sequences were: axial scanning mode, $\mathrm{kV}=130 \mathrm{kVp}, 100 \mathrm{~mA}, \mathrm{~N}=1 \mathrm{~mm}$ (number of slice) and $\mathrm{T}=5$

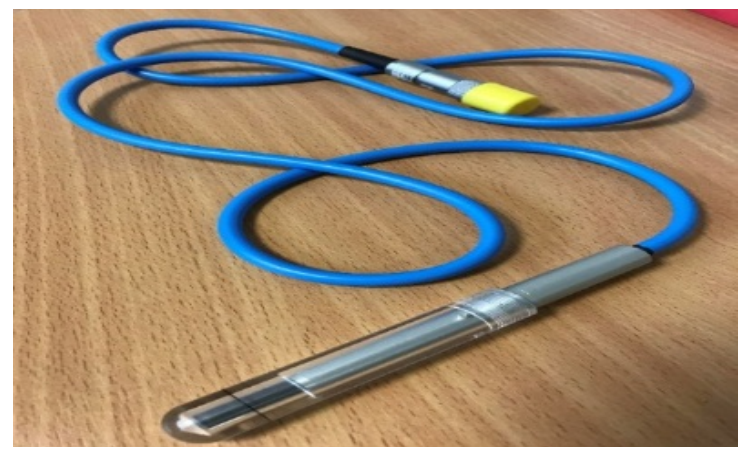

Figure 2. PTW pharmer champers. 


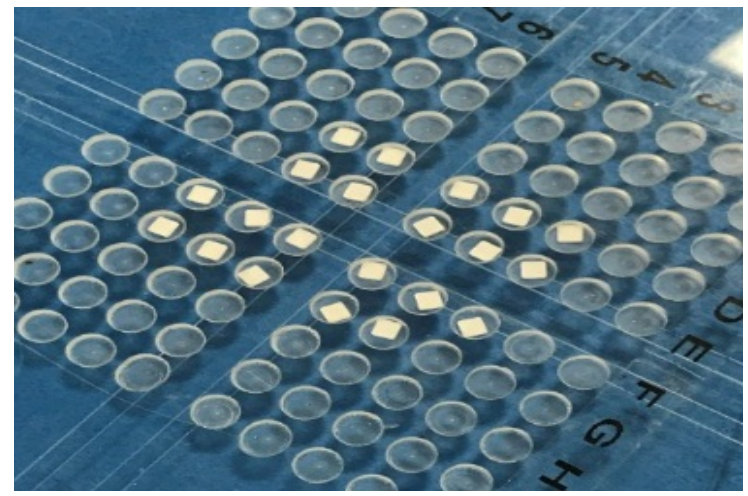

Figure 3. TLD 100H chips.

mm (tomographic section nominal width).

\subsubsection{Using Farmer Ionization Chamber}

1) Accumulated dose

Scanning a $D_{(\mathrm{eq})}$ phantom moving along the $\mathrm{z}$-axis through a rotating $\mathrm{x}$-ray produces an accumulated dose that can be envisaged as a dose amassed by superpositioning stationary, longitudinally displaced single-scan dose profiles.

With the small ion chamber method, the accumulated dose is directly measured by integrating the current from an ion chamber located at a fixed point in the phantom at the mid-point of the scanned length Figure 4 [11], while the $D_{\text {(eq) }}$ phantom central axis was aligned with the CT rotation axis. The farmer chamber was placed in the phantom in order to center the charge collection volume. The electrometer, which was placed outside the scan room, was connected to the farmer chamber using a cable that reduced the extra chamber current. The charge collected in the ion chamber $q_{h}(\mathrm{nC})$ was converted to accumulated dose $D_{(0)}$ as follows:

$$
D_{(0)}=N_{k} * q_{h} *\left(\mu_{(e n)} / \rho\right)_{\text {air }}^{\text {water }}[12]
$$

where $q_{h}(\mathrm{nC})$ is, the total charge collected by the ionization chamber during scanning over the length $L, N_{k}(\mathrm{mGy} / \mathrm{nC})$ is the ionization chamber calibration factor supplied by national standards of the German national laboratory,

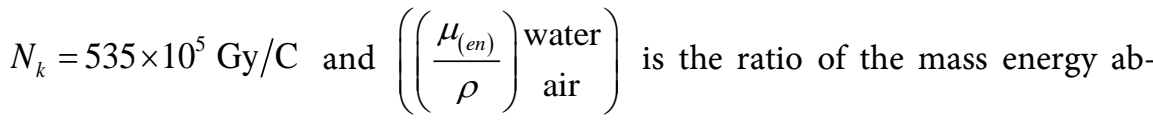
sorption coefficient for water to air. This ratio will vary somewhat with $\mathrm{kVp}$, with the phantom, between the central and peripheral axes and even as the ionization chamber moves along the z-axis. However, the variation is less than 3\% [8] [13]. By following the IAEA TRS-277 [10], the energy absorption coefficient ratio water to air was 1.036 .

The accumulated dose in center and peripheral locations was measured for frequently clinically used protocols [14]. The parameters of each protocol are detailed in Table 1. Protocol 1 is used for head and protocol 2 for chest. The third one is used for the abdomen. 

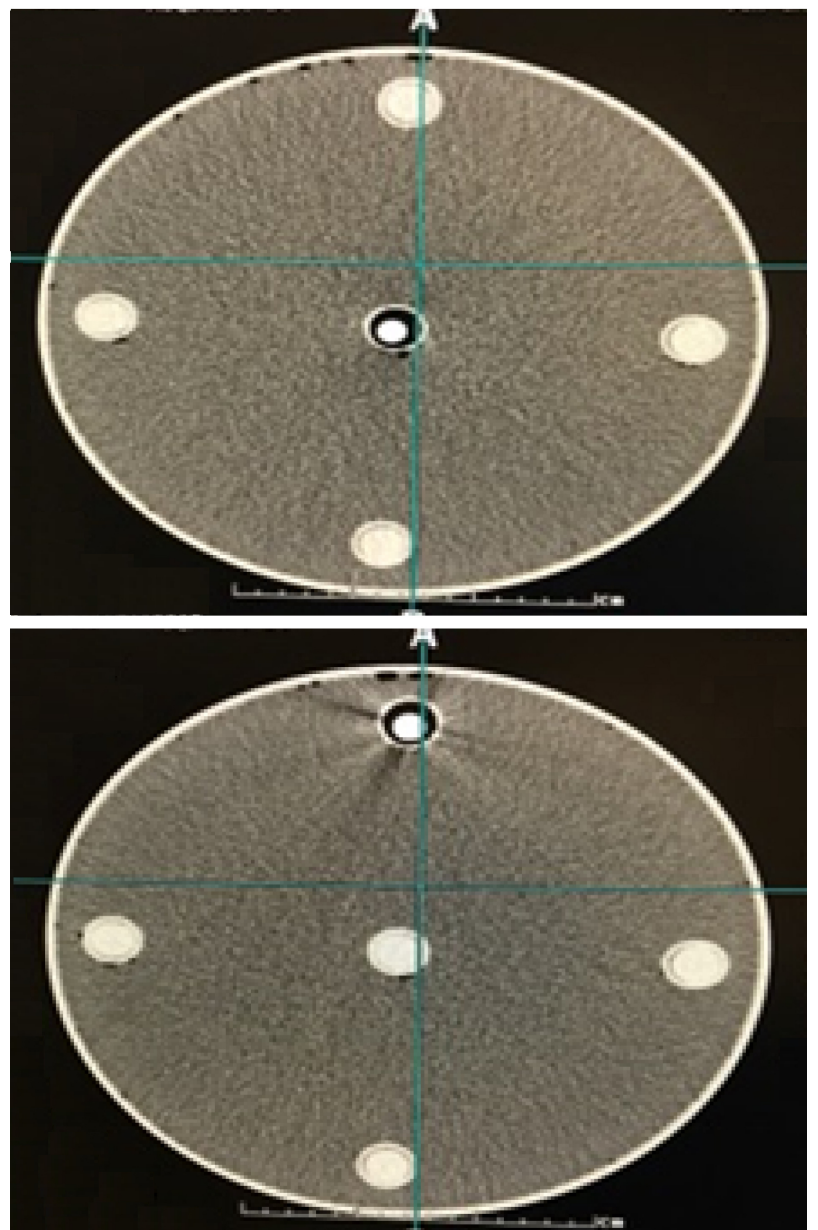

Figure 4. Farmer chamber position (center and peripheral).

Table 1. Details for the sequence of the protocols used. $(\mathrm{n}=$ slice number, $\mathrm{T}=$ slice width, $\mathrm{b}=$ table increment in a sequence of axial scanning or continuous table advance per rotation during helical scanning).

\begin{tabular}{cccc}
\hline \multirow{2}{*}{ Protocol } & Protocol 1 & Protocol 2 & Protocol 3 \\
\cline { 2 - 4 } & Head & Chest & Abdomen \\
\hline Scanning mode & Axial & Axial & Axial \\
KVp & 130 & 120 & 130 \\
mA & 100 & 100 & 100 \\
N (mm) & 2 & 2 & 2 \\
T (mm) & 5 & 5 & 4 \\
Pitch factor $=$ b/nT & 1 & 1 & 1 \\
Time per tube rotation & $1 \mathrm{~s}$ & $1 \mathrm{~s}$ & $1 \mathrm{~s}$ \\
\hline
\end{tabular}

\section{2) Equilibrium dose}

The equilibrium dose is based on an upper limiting value that is derived from the relationship of the scanning length and cumulative dose. When the scanning length $(L)$ increases, the cumulative dose at $z=0$ increases along with accumulating contributions from the (scatter tails) outlying scan sections, toward an 
upper limiting value in which the source of scatter radiation only makes negligibly small additional contributions. The equilibrium dose $D_{(\mathrm{eq})}$ is given by:

$$
D_{\text {eq }}=\frac{D_{(0)}}{h_{(L)}}[8][15]
$$

The $h_{(L)}$ is approach to equilibrium function, where $h_{(L)}=1$ when $L$ becomes large enough to yield scatter equilibrium at $z_{-} 0 . h_{(L)}$ has an approximate form as a constant plus an exponentially dependent term leading to saturation [8]. The equilibrium dose was determined for both the centre and peripheral axes for three protocols (Table 1) then the volume average equilibrium dose [8] [14] was measured by Equation (3) and compared with the CTDI volume.

$$
D_{\text {eq }}=\frac{1}{2} D_{\text {eq,center }}+\frac{1}{2} D_{\text {eq,peripheralr }}[14][15]
$$

3) Using pencil ionization chamber

Computed tomography dose index concepts are well documented and it is the primary dose measurement in CT scan currently [16] [17]. Computed tomography dose index (CTDI) represents the average absorbed dose, along the $\mathrm{z}$-axis, from a series of contiguous irradiations. It is measured from one axial CT and is calculated by dividing the integrated absorbed dose by the nominal total beam collimation [17] [18].

$$
\text { CTDI }=\frac{1}{N T} \int_{-\infty}^{\infty} D(Z) \mathrm{d} z \quad[16][19]
$$

where $D(Z)$ the radiation dose profile along the $z$-axis, $N$ the number of tomographic sections imaged in a single axial scan and $T$ the number of data channels.

The CTDI was proposed and established in the USA by the Food and Drug Administration (FDA) [20] [21]. It is measured by using $100 \mathrm{~mm}$ long ionization chamber and determined by

$$
\mathrm{CTDI}_{100}(\mathrm{~Gy})=\frac{100 \mathrm{~mm} \cdot \text { meter reading }(\mathrm{Gy})}{N T(\mathrm{~mm})} \text { [16] [19] [22] }
$$

The CTDI 100 was determined for central and peripheral axes for three protocols using the Perspex phantom. To make a comparison between the CTDI approach and dose equilibrium method, we utilized the approach of dose equilibrium, by taking the dose from the pencil chamber for one slice and multiplied by 100 then dividing by number of slices and the thickness (NT) of the beam (using Equation (5)) determining the dose profile over the whole chamber. This allows the assessment of CTDI volume and allows the comparison of it with the volume average equilibrium dose.

\subsubsection{Using TLD-100H}

Dose measurements were also performed in the $D_{(\mathrm{eq})}$ phantom using thermoluminescent dosimeters (TLD-100H) for the verification of the dose calculation by farmer chamber (dose equilibrium method). TLD-100H chips have a high sensi- 
tivity and they are commonly used in diagnostic radiology. However, they have different annealing and reading requirements than other TLDs. TLD-100H chips must not exceed a temperature of $240^{\circ} \mathrm{C}$ and the recommended annealing cycle for TLD- $100 \mathrm{H}$ chips are $240^{\circ} \mathrm{C}$ for 10 minutes [23].

Twenty chips of TLD-100H were calibrated with a diagnostic X-ray system. The luminescent signals were measured by an automated TLD reader (Model 3500, Harshaw), the calibration factor for the TLDs was determined by using the signal integration of the last two peak [10], the calibration factor was 0.00385 $\mathrm{mGy} / \mathrm{nC}$. Three of TLDs chips where excluded as they were found to be two standard deviation away from a mean.

Four TLD chips Figure 5 were placed in the center of $D_{(\mathrm{eq})}$ phantom for each protocol, and the average of four readings for each protocol is reported and compared with dose values obtained from the farmer chamber.

\section{Results and Discussion}

\subsection{Beam Attenuation in the $D_{(\mathrm{eq})}$ Phantom and CTDI Phantom}

The dose outputs from the $D_{\text {(eq) }}$ phantom and the Perspex phantom are $0.34 \pm$ $0.01 \mathrm{mGy}$ and $0.33 \pm 0.01 \mathrm{mGy}$ respectively as shown in Table 2 . Thus, the dose attenuation in $D_{\text {(eq) }}$ phantom is equivalent to the dose attenuation on any standard CTDI phantom.

\subsection{The Volume Average Equilibrium Dose}

The volume average equilibrium dose is obtained by using Equation (3). The central and peripheral were measured for three protocols to find the volume average equilibrium dose, which listed in Table 3 . The volume average equilibrium

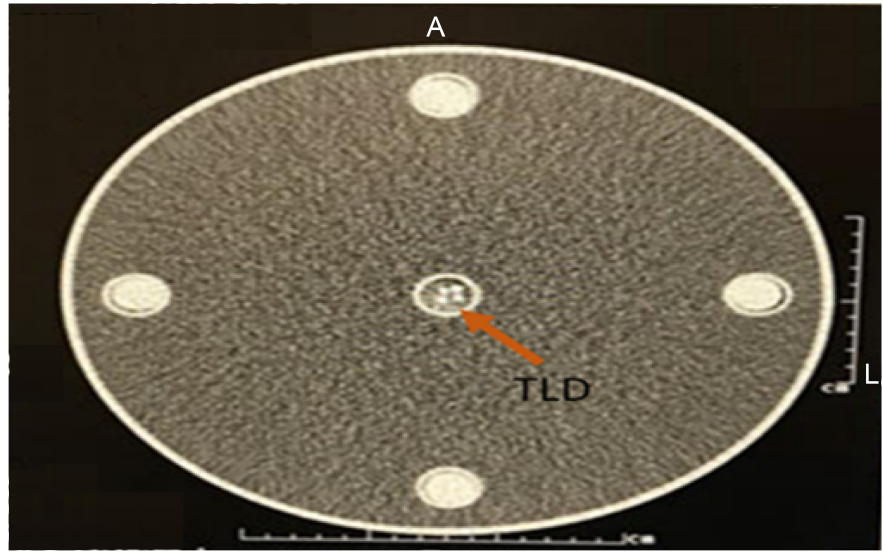

Figure 5. TLD chips in the center of $D_{(\mathrm{eq})}$ phantom.

Table 2. Single slice dose output at the center of $\mathrm{D}_{(\mathrm{eq})}$ and Perspex phantoms.

\begin{tabular}{ccccc}
\hline Phantom & First reading & Second reading & Third reading & Average \\
\hline Water phantom & 0.33 & 0.35 & 0.34 & $0.34 \pm 0.01$ \\
Perspex phantom & 0.33 & 0.34 & 0.33 & $0.33 \pm 0.01$ \\
\hline
\end{tabular}


Table 3. Volume average equilibrium dose.

\begin{tabular}{ccccccc}
\hline Protocols & $\begin{array}{c}\text { Center } \\
(\mathrm{mG})\end{array}$ & Average $\pm \mathrm{SD}$ & $\begin{array}{c}\text { Peripheral } \\
(\mathrm{m} \text { ean) }(\mathrm{mGy})\end{array}$ & Average $\pm \mathrm{SD}$ & $\begin{array}{c}D_{(\mathrm{eq})} \\
(\mathrm{mG})\end{array}$ & Average \pm SD \\
\hline \multirow{3}{*}{ Head } & 12.49 & & 14.91 & & 13.70 & \\
& 12.73 & $12.63 \pm 0.05$ & 14.83 & $14.94 \pm 0.13$ & 13.78 & $13.79 \pm 0.0 .06$ \\
& 12.68 & & 15.10 & & 13.89 & \\
& 10.45 & & 12.55 & & 11.51 & \\
Chest & 10.64 & $10.58 \pm 0.09$ & 12.41 & $12.53 \pm 0.0 .09$ & 11.52 & $11.56 \pm 0.0 .06$ \\
& 10.65 & & 12.65 & & 11.65 & \\
& 10.12 & & 13.31 & & 11.71 & \\
Abdomen & 10.10 & $10.04 \pm 0.09$ & 13.22 & $13.22 \pm 0.0 .06$ & 11.66 & $11.63 \pm 0.0 .07$ \\
& 9.91 & & 13.15 & & 11.53 & \\
Average & & 11.08 & & 13.56 & & 12.32 \\
\hline
\end{tabular}

dose was $12.3 \mathrm{mGy}$ for all protocols.

\subsection{CTDI Volume Measurements and Comparison with the Volume Average Equilibrium Dose}

The CTDI volume was measured by a Perspex phantom for all protocols and compared to the volume average equilibrium doses for head, chest and abdomen, resulting in an underestimation of $32 \%, 35 \%$ and $25 \%$ respectively across the three protocols. This data is presented in Table 4. Additionally, the measured single slice dose profile at the center of the $D_{(\text {eq) }}$ phantom Figure 6 indicated that the scan length of $100 \mathrm{~mm}$ was not long enough to measure all the tails of the scattered dose distribution, confirming that the CTDI methodology is no longer adequate to accurately characterise $\mathrm{CT}$ dose performance.

\subsection{TLD Measurement and Comparison with Equilibrium Dose}

The comparison between the TLD in center Figure 5 and dose equilibrium in center is shown in Table 5. The variation between the TLD and equilibrium dose for all protocols was less than 5\%; therefore, TLD confirmed the accuracy of the dose equilibrium methodology.

\section{Conclusions}

Organ and tissue radiation dose prior to a CT scan is currently estimated in Ireland's health service using the IMPACT software [24] and other healthcare system use similar estimates. These estimates are based on detailed system specific Monte Carlo simulations and a mathematical model of human phantoms [9].

The organ dose and accumulative dose are based on CT dose indexes (CTDI), which we have shown to underestimate the dose. As can be seen (Table 4), the $10-\mathrm{cm}$ chamber measurement underestimates the actual dose by $25 \%$ to $35 \%$. 
Table 4. CTDI volume compared with volume average equilibrium dose.

\begin{tabular}{cccccc}
\hline Protocols & Deq $(\mathrm{mGy})$ & average \pm SD & $\begin{array}{c}\text { CTDI } \\
\text { volume } \\
(\mathrm{mGy})\end{array}$ & average \pm SD & Variation \\
\hline \multirow{2}{*}{ Head } & 13.70 & & 10.4 & & \\
& 13.78 & $13.79 \pm 0.0 .06$ & 10.37 & $10.38 \pm 0.01$ & $32 \%$ \\
& 13.89 & & 10.39 & & \\
Chest & 11.51 & & 8.57 & & \\
& 11.52 & $11.56 \pm 0.0 .06$ & 8.49 & $8.54 \pm 0.03$ & \\
& 11.65 & & 8.57 & & $25 \%$ \\
& 11.71 & & 9.26 & & \\
\hline \multirow{2}{*}{ Abdomen } & 11.66 & $11.63 \pm 0.0 .07$ & 9.20 & $9.25 \pm 0.03$ & \\
& 11.53 & & 9.29 & & \\
\hline
\end{tabular}

Table 5. TLD compared with equilibrium dose.

\begin{tabular}{|c|c|c|c|c|c|}
\hline Protocols & $\begin{array}{c}\text { Deq } \\
\text { Center (mGy) }\end{array}$ & average \pm SD & $\begin{array}{l}\text { TLD } \\
\text { center }\end{array}$ & average \pm SD & Variation \\
\hline \multirow{4}{*}{ Head } & 12.49 & \multirow{4}{*}{$12.63 \pm 0.0 .05$} & 12.86 & \multirow{4}{*}{$12.93 \pm 0.47$} & $2 \%$ \\
\hline & 12.73 & & 13.68 & & \\
\hline & 12.68 & & 12.36 & & \\
\hline & & & 12.84 & & \\
\hline \multirow{4}{*}{ Chest } & 10.45 & \multirow{4}{*}{$10.58 \pm 0.0 .09$} & 10.20 & \multirow{4}{*}{$11.04 \pm 0.52$} & $4 \%$ \\
\hline & 10.64 & & 11.42 & & \\
\hline & 10.65 & & 11.54 & & \\
\hline & & & 11.01 & & \\
\hline \multirow{4}{*}{ Abdomen } & 10.1 & \multirow{4}{*}{$10.04 \pm 0.0 .09$} & 9.73 & \multirow{4}{*}{$10.42 \pm 0.53$} & $3 \%$ \\
\hline & 10.1 & & 10.89 & & \\
\hline & 9.91 & & 10.99 & & \\
\hline & & & 10.08 & & \\
\hline
\end{tabular}

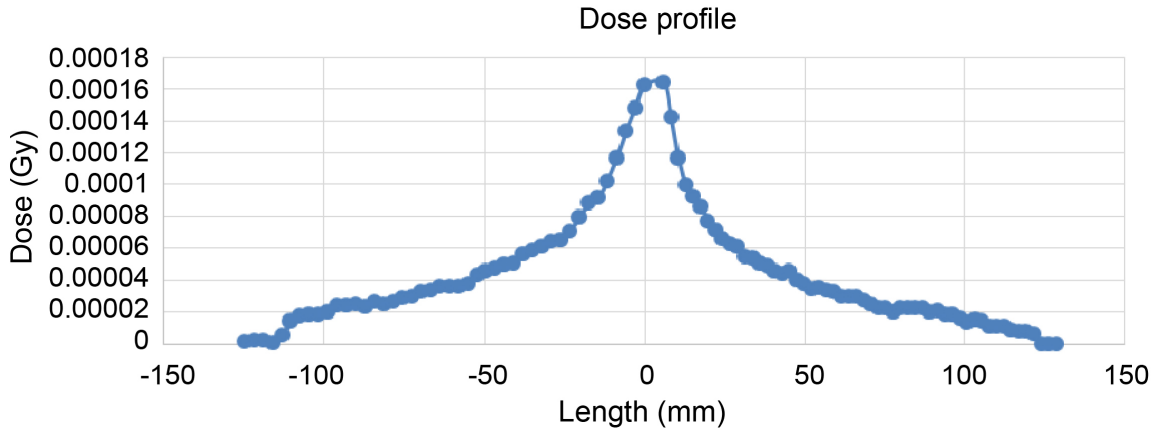

Figure 6. Single slice dose profile at the center.

Descamps et al. [14], determined the volume equilibrium doses for chest, prostate and metastasis protocols to be $12.3 \mathrm{mGy}, 12.3 \mathrm{mGy}$ and $12.5 \mathrm{mGy}$, respec- 
tively. This was compared to the CTDI volumes for the same three protocols, which were 9.1 mGy, 9.3 mGy and 9.3 mGy, respectively. They reported underestimation of $30 \%$ to $35 \%$ in dose as measured by CTDI. Furthermore, Dixon et al. [25], found that the current methodology based on the measurement of the integral of the single slice profile using a $10 \mathrm{~cm}$ long ion chamber is underestimate the equilibrium dose and dose line integral by about $20 \%$. Thus the two studies are in good agreement with our findings.

The $100-\mathrm{mm}$ pencil chamber is too short and it underestimates the limiting equilibrium dose for any scan length above $100 \mathrm{~mm}$. As a result, the tissue weighted organ dose obtained from IMPACT software may underestimate the radiation dose absorbed by the organs, increasing patient risk.

Alternately, using an ion chamber shorter than $1 \mathrm{~cm}$, and a phantom long enough to establish dose equilibrium in the center we can provide a more realistic dose estimate. In addition, a measurement of accumulated dose can be determined at any point in the phantom not just at the center of the scan length which is the only location at which the pencil chamber in CTDI can measure accumulative dose to formalize a dose prediction. The dose equilibrium method is more flexible and not significantly more time consuming than the current method that uses a long chamber.

\section{Acknowledgements}

Stuart Harris NUIG for help constructing the phantom, Sinead Cleary and Michael O'Conner Galway University Hospital for organizing access and training on the CT system and Matthew Donoghue for organizing access to a CTDI phantom and pencil ion chamber. The authors would also like to acknowledge the input of Prof Van der Putten who has sadly passed away.

\section{References}

[1] IMV (2012) CT Market Outlook Report. IMV Medical Information Division, Des Plaines.

[2] NCRP (2009) Ionizing Radiation Exposure of the Population of the United States. NCRP Report No. 160, National Council on Radiation Protection and Measurements, Bethesda.

[3] Tian, X., Li, X., Segars, W.P., Frush, D.P., Paulson, E.K. and Samei, E. (2013) Dose Coefficients in Pediatric and Adult Abdominopelvic CT Based on 100 Patient Models. Physics in Medicine and Biology, 58, 8755-8768. https://doi.org/10.1088/0031-9155/58/24/8755

[4] Celina, L., Yogesh, T. and Nancy, L. (2017) Dose Comparison between CTDI and the AAPM Report No. 111 Methodology in Adult, Adolescent, and Child Head Phantom. Physics of Medical Imaging, 101320T.

[5] Huda, W. and Mettler, F.A. (2011) Volume CT Dose Index and Dose-Length Product Displayed during CT: What Good Are They? Radiology, 258, 236-242. https://doi.org/10.1148/radiol.10100297

[6] Cinthia, P., Divanizia, S. and Luiz, S. (2016) Comparison of Dose Measurements in CT Using a Photodiode and a Small Ion Chamber. Radiation Measurements, 91, 
50-53.

[7] Dixon, R.L. (2006) Restructuring CT Dosimetry-A Realistic Strategy for the Future Requiem for the Pencil Chamber. Medical Physics, 33, 3973-3976.

https://doi.org/10.1118/1.2336504

[8] AAPM (2010) Report of AAPM Task Group 111: The Future of CT Dosimetry: Comprehensive Methodology for the Evaluation of Radiation Dose in X-Ray Computed Tomography. AAPM Report No. 111, American Association of Physicist in Medicine, City Park.

[9] Matsubara, K. (2017) Computed Tomography Dosimetry: From Basic to State-ofthe-Art Techniques. Medical Physics, 5.

[10] IAEA (1992) Review of Data and Methods Recommended in the International Code of Practice, IAEA TECDOC 897. IAEA Technical Reports Series 277, Absorbed Dose Determination in Photon and Electron Beams, IAEA, Vienna.

[11] Trevisan, D., Ravanelli, D. and Valentini, A. (2014) Measurements of Computed Tomography Dose Index for Clinical Scans. Radiation Protection Dosimetry, 158, 389-398. https://doi.org/10.1093/rpd/nct236

[12] Dixon, R.L. and Ballard, A.C. (2007) Experimental Validation of a Versatile System of CT Dosimetry using a Conventional Ion Chamber: Beyond CTDI100. Medical Physics, 34, 3399-3413. https://doi.org/10.1118/1.2757084

[13] Zhou, H. and Boone, J.M. (2008) Monte Carlo Evaluation of CTDI in Infinitely Long Cylinders of Water, Polyethylene and PMMA with Diameters from $10 \mathrm{~mm}$ to 500 mm. Medical Physics, 35, 2424-2431. https://doi.org/10.1118/1.2921829

[14] Descamps, C., Gonzalez, M., Garrigo, E., Germanier, A. and Venencia, D. (2012) Measurements of the Dose Delivered during CT Exams using AAPM Task Group Report No. 111. Journal of Applied Clinical Medical Physics, 13, 3934-3942. https://doi.org/10.1120/jacmp.v13i6.3934

[15] Maria, C., Marcia, S. and Ricardo, T. (2016) CTDI versus New AAPM Metrics to Assess Doses in CT: A Case Study. Brazilian Journal of Radiation Sciences, 4, 1-15.

[16] White, R. (1977) The Formulation of Tissue Substitute Materials using Basic Interaction Data. Physics in Medicine \& Biology, 22, 889-899.

https://doi.org/10.1088/0031-9155/22/5/008

[17] IEC (2010) Amendment 1 to IEC 60601-2-44: 2009 Medical Electrical Equipment: Parts 2-44. Particular Requirements for the Basic Safety and Essential Performance of X-Ray Equipment for Computed Tomography Committee Draft (CD) 62B/804/ CD. International Electrotechnical Commission, Geneva.

[18] Kalender, W.A. (2011) Computed Tomography: Fundamentals, System Technology, Image Quality, Applications. 3rd Edition, Publicis, Erlangen.

[19] Alaraky, R. (2015) Measurement of Radiation Dose for Patients by CT Scan and X-Rays. Doctoral Dissertation, Sudan University of Science and Technology, Khartoum.

[20] IAEA (2011) Status of Computed Tomography Dosimetry for Wide Cone Beam Scanners. Atomic Energy Agency, Vienna.

[21] Shope, T.B., Gagne, R.M. and Johnson, G.C. (1981) A Method for Describing the Doses Delivered by Transmission X-Ray Computed Tomography. Medical Physics, 8, 488-495. https://doi.org/10.1118/1.594995

[22] Bakalayar, D.M. (2006) A Critical Look at the Numerical Coefficients in CTDIVOL. Medical Physics, 33, 2003. https://doi.org/10.1118/1.2240267

[23] QADOS. Data Sheet for Harshaw TLD Model 3500 Materials and Assemblies. 
http://www.qados.co.uk

[24] Willi, K. (2014) Dose in X-Ray Computed Tomography. Physics in Medicine \& Biology, 59, R129-R150. https://doi.org/10.1088/0031-9155/59/3/R129

[25] Dixon, R.L. (2003) A New Look at CT Dose Measurement: Beyond CTDI. Medical Physics, 30, 1272-1280. https://doi.org/10.1118/1.1576952 